Received: 28.02 .2018

Revised: 12.04.2018

Accepted: 20.04.2018

DOI: $10.17804 / 2410-9908.2018 .2 .092-109$

\title{
EXACT SOLUTIONS FOR A COUETTE-HIEMENZ CREEPING CONVECTIVE FLOW WITH LINEAR TEMPERATURE DISTRIBUTION ON THE UPPER BOUNDARY
}

\author{
V. V. Privalova ${ }^{\text {a)* }}$, E. Yu. Prosviryakov ${ }^{\text {b) }}$ \\ Institute of Engineering Science, Ural Branch of the Russian Academy of Sciences, \\ 34 Komsomolskaya St., Ekaterinburg, 620049, Russian Federation \\ a) iD http://orcid.org/0000-0002-8648-0900 valentprival@gmail.com; \\ b) iD http://orcid.org/0000-0002-2349-7801 《evgen_pros@ mail.ru \\ *Corresponding author. E-mail: valentprival@gmail.com \\ Address for correspondence: 34, Komsomolskaya st., Ekaterinburg, 620049, Russian Federation \\ Tel.: +7 (343) 37535 76; Fax: 3745330
}

A new exact solution is found for the plane convection of a viscous incompressible fluid describable by the Oberbeck-Boussinesq equation system in an infinite thin layer. The solution of the boundary-value problem is obtained for the fluid flow arising in the case of inhomogeneous velocity distribution and a linear heat source at the upper boundary of an infinite layer of a viscous incompressible fluid. A creeping convective flow is studied by the generalization of the isothermal class of the Hiemenz exact solutions. The temperature and pressure fields are linear with respect to the horizontal coordinate in this class of solutions. The analysis of polynomial solutions describing natural fluid convection in the Stokes approximation is presented. The paper shows the existence of points where the velocity field vanishes inside the fluid layer. These points are termed stagnation points and indicate the presence of counterflows in the fluid. Similar investigations are carried out for the temperature and pressure fields. The isotherms and isobars are shown to be always locally parabolic or hyperbolic, i.e. to have an infinitely distant point, due to the structure of the discussed exact solution.

Keywords: counterflow, exact solution, Couette flow, Hiemenz flow, Oberbeck-Boussinesq equation, Stokes approximation, stagnation point, stratification.

\section{References}

1. Couette M. Etudes sur le frottement des liquids. Ann. Chim. Phys., 1890, vol. 21, pp. 433-510.

2. Landau L.D., Lifshitz E.M. Course of Theoretical Physics. Vol. 6. Fluid mechanics. Pergamon Press, Oxford, 1987, 539 p.

3. Drazin P.G. The Navier-Stokes equations: A classification of flows and exact solutions. Cambridge, Cambridge Univ. Press, 2006, 196 p.

4. Boronin S.A. Stability of the plane Couette flow of a disperse medium with a finite volume fraction of the particles. Fluid Dynamics, 2011, vol. 46, pp. 64-71.

DOI: $10.1134 / \mathrm{S} 0015462811010078$.

5. Georgiyevskii D.V. Generalized Joseph Estimates of Stability of Plane Shear Flows with Scalar Nonlinearity. Bulletin of the Russian Academy of Sciences: Physics, 2011, vol. 75, no. 1, pp. 149-152. DOI: $10.3103 /$ S1062873810121044.

6. Zhuk V.I., Protsenko I.G. Asymptotic model for the evolution of perturbations in the plane Couette-Poiseuille flow. Doklady Mathematics, 2006, vol. 74, no. 3, pp. 896-900. DOI: $10.1134 / \mathrm{S} 1064562406060287$. 
7. Rudyak V.Ya., Isakov E.B., Bord E.G. Instability of plane Couette flow of two-phase liquids. Technical Physics Letters, 1998, vol. 24, pp. 199-200. DOI: 10.1134/1.1262051.

8. Troshkin O.V. Nonlinear stability of Couette, Poiseuille and Kolmogorov plane channel flows. Doklady Mathematics, 2012, vol. 85, no. 2, pp. 181-185. DOI: 10.1134/S1064562412020068.

9. Shalybkov D.A. Hydrodynamic and hydromagnetic stability of the Couette flow. PhysicsUspekhi, 2009, vol. 52, no. 9, pp. 915-935. DOI: 10.3367/UFNe.0179.200909d.0971.

10. Abramyan A.K., Mirantsev L.V., Kuchmin A.Yu. Modeling of processes at Couette simple fluid flow in flat nano-scopic canal. Matematicheskoe Modelirovanie, 2012, vol. 24, no. 4, pp. 3-21 (In Russian).

11. Aristov S.N., Prosviryakov E.Yu. Nonuniform convective Couette flow. Fluid Dynamics, 2016, vol. 51, pp. 581-587. DOI: 10.1134/S001546281605001X.

12. Aristov S.N., Prosviryakov E.Yu. A new class of exact solutions for three-dimensional thermal diffusion equations. Theor. Found. Chem. Eng., 2016, vol. 50, no. 3, pp. 286-293. DOI: $10.1134 /$ S0040579516030027.

13. Babkin V. A. Plane Turbulent Couette Flow. Journal of Engineering Physics and Thermophysics, vol. 76, iss. 6, pp. 1251-1254. DOI: 10.1023/B:JOEP.0000012026.19646.c6.

14. Belyaeva N.A., Kuznetsov K.P. Analysis of a nonlinear dynamic model of the Couette flow for structured liquid in a flat gap. Vestn. Samar. Gos. Tekhn. Univ. Ser. Fiz.-Mat. Nauki, 2012, no. 2 (27), pp. 85-92. DOI: 10.14498/vsgtu1018. (In Russian).

15. Gavrilenko S.L., Shil'ko S.V., Vasin R.A. Characteristics of a viscoplastic material in the couette flow. Journal of Applied Mechanics and Technical Physics, 2002, vol. 43, iss. 3, pp 439-444. DOI: 10.1023/A:1015378622918.

16. Kudinov V.A., Kudinov I.V. Reception of exact analytical decisions of the hyperbolic equations of movement at a dispersed current of Kuetta. Izvestiya Rossiyskoy Akademii Nauk. Energeti$k a, 2012$, no. 1, pp. 119-133 (In Russian).

17. Malyshev V.A., Manita A.D. Stochastic Micromodel of the Couette Flow. Theory of Probability \& its Applications, 2009, vol. 53, iss. 4, pp. 716-727. DOI: 10.1137/S0040585X97983924.

18. Pukhnachev V.V., Pukhnacheva T.P. Couette Problem for Kelvin-Voigt Medium. Journal of Mathematical Sciences, 2012, vol. 186, iss. 3, pp. 495-510. DOI: 10.1007/s10958-012-1003-0.

19. Skulsky O.I., Aristov S.N. Mekhanika anomalno vyazkikh zhidkostey [Mechanics of QuasiViscous Fluids]. Moscow-Izhevsk, NITs Regularnaya i Khaoticheskaya Dinamika Publ., 2003, 156 p. (In Russian).

20. Aristov S.N., Privalova V.V., Prosviryakov E.Yu. Stationary nonisothermal Couette flow. Quadratic heating of the upper boundary of the fluid layer. Russian Journal of Nonlinear Dynamics, 2016, vol. 12, no. 2, pp. 167-178. DOI: 10.20537/nd1602001. (In Russian).

21. Aristov S.N., Prosviryakov E.Yu. On one class of analytic solutions of the stationary axisymmetric convection Bénard-Maragoni viscous incompreeible fluid. Vestn. Samar. Gos. Tekh. Univ., Ser. Fiz.-Mat. Nauki, 2013, no. 3 (32), pp. 110-118. DOI: 10.14498/vsgtu1205. (In Russian).

22. Aristov S.N. Prosviryakov E.Yu. Exact Solutions of Thermocapillary Convection in a Localized Heating of a Plane Layer of a Viscous Incompressible Fluid. Vestnik KGTU im. A.N. Tupoleva, 2014, no. 3, pp. 7-12. (In Russian).

23. Aristov S.N., Vlasova S.S., Prosviryakov E.Yu. Linear Benard-Marangoni convection with quadratic heating on top of a plane layer of a viscous incompressible fluid. Polzunovskiy Vestnik, 2014, no. 4-2, pp. 95-102. (In Russian).

24. Aristov S.N., Shvarts K.G. Convective heat transfer in a locally heated plane incompressible fluid layer. Fluid Dynamics, 2013, vol. 48, pp. 330-335. DOI: 10.1134/S001546281303006X.

25. Vlasova S.S., Prosviryakov E.Y. Parabolic convective motion of a fluid cooled from below with the heat exchange at the free boundary. Russian Aeronautics, 2016, vol. 59, no. 4, pp. 529-535. DOI: $10.3103 / \mathrm{S} 1068799816040140$. 
26. Vlasova S.S., Prosviryakov E.Yu. Two-dimensional convection of an incompressible viscous fluid with the heat exchange on the free border. Vestn. Samar. Gos. Tekh. Univ., Ser. Fiz.-Mat. Nauki, 2016, vol. 20, no. 3, pp. 567-577. DOI: 10.14498/vsgtu1483.

27. Hiemenz K. Die Grenzschicht an einem in den gleichförmigen Flüssigkeit-sstrom eingetauchten geraden Kreiszylinder. Dingler's Politech. J., 1911, vol. 326, pp. 321-324.

28. Andreev V.K., Cheremnykh E.N. 2D thermocapillary motion of three fluids in a flat channel. Journal of Siberian Federal University. Mathematics and physics, 2016, vol. 9, issue. 4, pp. 404-415. DOI: 10.17516/1997-1397-2016-9-4-404-415.

29. Andreev V.K., Cheremnykh E.N. The joint creeping motion of three viscid liquids in a plane layer: A priori estimates and convergence to steady flow. Journal of Applied and Industrial Mathematics, 2016, vol. 10, no. 1, pp. 7-20. DOI: 10.1134/S1990478916010026.

30. Andreev V.K., Sobachkina N.L. Dvizhenie binarnoy smesi v ploskikh i tsilindricheskikh oblastyakh [The Motion of a Binary Mixture in Planar and Cylindrical Regions]. Krasnoyarsk, SFU Publ., 2012, 187 p. (In Russian).

31. Aristov S.N., Knyazev D.V., Polyanin A.D. Exact solutions of the Navier-Stokes equations with the linear dependence of velocity components on two space variables. Theoretical Foundations of Chemical Engineering, 2009, vol. 43, no. 5, pp. 642-662.

DOI: 10.1134/S0040579509050066.

32. Pukhnachev V.V. Group Properties of the Navier-Stokes Equations in a Plane Case. Prikl. Mekh. Tekh. Fiz., 1960, no. 1, pp. 83-90.

33. Ekman V.W. On the Influence of the Earth's Rotation on Ocean-Currents. Ark. Mat. Astron. Fys., 1905, vol. 2, no. 11, pp. 1-52.

34. Gershuni G.Z., Zhukhovitskii E.M. Convective Stability of Incompressible Fluids. Israel Program for Scientific Translations. Jerusalem: Keter Publishing House, 1976, 330 p.

35. Andreev V.K., Gaponenko Ya.A., Goncharova O.N., Pukhnachev V.V. Mathematical Models of Convection. Berlin, Boston: De Gryuter Publ., 2012, 417 p. DOI: 10.1515/9783110258592.

36. Sidorov A.F. Two classes of solutions of the fluid and gas mechanics equations and their connection to traveling wave theory. J. Appl. Mech. Tech. Phys., 1989, vol. 30, no. 2, pp. 197-203. DOI: $10.1007 / \mathrm{BF} 00852164$.

37. Lin C.C. Note on a class of exact solutions in magneto-hydrodynamics. Arch. Rational Mech. Anal., 1958, vol. 1, pp. 391-395. 
Подана в журнал: 28.02.2018

УДК 539

DOI: $10.17804 / 2410-9908.2018 .2 .092-109$

\title{
ТОЧНЫЕ РЕШЕНИЯ, ОПИСЫВАЮЩИЕ КОНВЕКТИВНОЕ ПОЛЗУЩЕЕ ТЕЧЕНИЕ КУЭТТА-ХИМЕНЦА ПРИ ЛИНЕЙНОМ РАСПРЕДЕЛЕНИИ ТЕМПЕРАТУРЫ НА ВЕРХНЕЙ ГРАНИЦЕ
}

\author{
В. В. Привалова ${ }^{\text {a)* }}$ Е. Ю. Просвиряков ${ }^{\text {) }}$ \\ Институт машиноведения Уральского отделения Российской академии наук, \\ ул. Комсомольская, 34, Екатеринбург, Российская Федерация \\ a) (ID http://orcid.org/0000-0002-8648-0900 ख valentprival@gmail.com; \\ б) (iD http://orcid.org/0000-0002-2349-7801 evgen_pros@ mail.ru \\ "Ответственный автор. Электронная почта: valentprival@gmail.com \\ Адрес для переписки: ул. Комсомольская, 34, Екатеринбург, Российская Федерация \\ Тел.: +7 (343) 375-35-76; факс: 374-53-30
}

Найдено новое точное решение плоской конвекции вязкой несжимаемой жидкости, описываемой системой уравнений Обербека-Буссинеска в бесконечном тонком слое. Решение краевой задачи получено для течения жидкости, возникающее при неоднородном распределении скоростей и линейного источника тепла на верхней границе бесконечного слоя вязкой несжимаемой жидкости. Исследование ползущего конвективного течения осуществляется посредством обобщения изотермического класса точных решений Хименца, в котором поля температуры и давления линейны относительно горизонтальной координаты. Приведен анализ полиномиальных решений, описывающих естественную конвекцию жидкости в приближении Стокса. Показано существование точек, в которых поле скоростей обращается в нуль внутри слоя жидкости, что определяет существование застойных точек и противотечений в жидкости. Аналогичные исследования проведены для полей температуры и давления. Показано, что изотермы и изобары из-за структуры рассматриваемого точного решения всегда будут локально параболическими или гиперболическими (иметь бесконечно удаленную точку).

Ключевые слова: противотечение, точное решение, течение Куэтта, течение Хименца, уравнение Обербека-Буссинеска, приближение Стокса, застойная точка, стратификация.

\section{1. Введение}

В самых разных теоретических и прикладных научных дисциплинах при решении технических задач часто используется модельное представление конвективного течения жидкости, движущейся между двумя бесконечными недеформируемыми пластинами с заданными скоростями. Если на границах слоя вязкой несжимаемой жидкости задаются скорости, то при изотермическом течении поле скоростей описывается точным решением Куэтта [1]. При изложении данного точного решения используют два подхода. В пользу первого способа представления течения Куэтта говорят, что это решение получено с использованием приближения Стокса для уравнений Навье-Стокса о преобладании вязких сил над инерционными эффектами, возникающими в движущейся жидкости [2]. В пользу второго подхода отметим, что решение Куэтта удовлетворяет нелинейным уравнениям Навье-Стокса, обращая при этом тождественно нулю конвективные (инерционные) слагаемые [1, 3].

С одной стороны, замеченная двойственность при интерпретации течения Куэтта позволяет исследовать нелинейные свойства течения Куэтта в задачах теории устойчивости изотермических течений вязкой несжимаемой жидкости [4-9]. С другой стороны, решение Ку- 
этта является первым примером стационарного ползущего течения вязкой несжимаемой жидкости, модификации которого находят применение в практических задачах, для которых также существует необходимость поиска точного решения нелинейной системы ОбербекаБуссинеска, а также в приближении Стокса, описывающей конвективное движение несжимаемой жидкости, обладающей диссипативными эффектами [11-26].

В настоящей статье сделана попытка обобщить установившееся классическое решение Куэтта для случая ползущих течений неизотермических вязких несжимаемых жидкостей. Следует отметить, что говоря о течении Куэтта при конвективном перемешивании жидкости, речь прежде всего идет о граничных условиях, задаваемых в виде скоростей на одной или обеих границах бесконечного слоя. Как было показано, в статьях $[11,18-21]$ для описания ползущих конвективных потоков вместо класса решений Куэтта необходимо использовать точное решение Хименца [27] с дальнейшей линеаризацией уравнений ОбербекаБуссинеска. Использование класса Хименца позволяет учесть не только горизонтальную скорость, но и распространение импульсов в поперечном направлении. Кроме того, модификации течения Хименца с успехом применяются в гидродинамике неизотермических высоковязких многослойных жидкостей [28-30].

Отдельный интерес вызывает эффект существования в слое жидкости застойных точек (точек покоя), вызванных учетом горизонтальных градиентов функций скорости, температуры и давления (стратификации в продольном направлении). Впервые точное решение, учитывающее эффект застойных точек при течении жидкости, было исследовано Хименцем $[27,31,32]$. Аналогичные эффекты в океанологии для вращающихся жидкостей были исследованы на начальных этапах Экманом [33]. Течение Экмана было рассмотрено как сдвиговое, т. е. вертикальная компонента скорости тождественно равнялась нулю.

В настоящей работе рассмотрены и проанализированы условия существования застойных точек для заданного теплового источника на верхней границе бесконечного слоя жидкости в зависимости от значений граничных условий. Приведены критерии существования противотечений и условия стратификации полей температуры и давления.

\section{2. Постановка задачи}

Рассматриваются уравнения плоского движения вязкой несжимаемой жидкости в бесконечном слое, описывающие влияние температуры на распределение гидродинамических полей, в приближении Буссинеска [2, 34]:

$$
\begin{gathered}
\frac{\partial V_{x}}{\partial t}+V_{x} \frac{\partial V_{x}}{\partial x}+V_{z} \frac{\partial V_{x}}{\partial z}=-\frac{\partial P}{\partial x}+v\left(\frac{\partial^{2} V_{x}}{\partial x^{2}}+\frac{\partial^{2} V_{x}}{\partial z^{2}}\right) \\
\frac{\partial V_{z}}{\partial t}+V_{x} \frac{\partial V_{z}}{\partial x}+V_{z} \frac{\partial V_{z}}{\partial z}=-\frac{\partial P}{\partial z}+v\left(\frac{\partial^{2} V_{z}}{\partial x^{2}}+\frac{\partial^{2} V_{z}}{\partial z^{2}}\right)+g \beta T \\
\frac{\partial T}{\partial t}+V_{x} \frac{\partial T}{\partial x}+V_{z} \frac{\partial T}{\partial z}=\chi\left(\frac{\partial^{2} T}{\partial x^{2}}+\frac{\partial^{2} T}{\partial z^{2}}\right) \\
\frac{\partial V_{x}}{\partial x}+\frac{\partial V_{z}}{\partial z}=0
\end{gathered}
$$

Здесь $V_{x}$ и $V_{z}$ - скорости параллельные, соответственно, координатным осям $O x$ и $O z$ прямоугольной декартовой системы координат; $P$ - отклонение давления от гидростатического, отнесенное к постоянной средней плотности жидкости $\rho ; T$ - отклонение от средней температуры; $v, \chi$ - коэффициенты кинематической вязкости и температуропро- 
водности жидкости соответственно; $g$ - ускорение свободного падения; $\beta$ - температурный коэффициент объемного расширения жидкости $[2,34]$.

Точное решение системы Обербека-Буссинеска (1) будем искать для стационарного случая в виде [12, 20, 31, 35-37]:

$$
\begin{gathered}
V_{x}(z)=U(z)+u_{1}(z) x, V_{z}(z)=w(z) ; \\
P(x, z)=P_{0}(z)+P_{1}(z) x ; \\
T(x, z, t)=T_{0}(z, t)+T_{1}(z, t) x .
\end{gathered}
$$

Поля давления, температуры и горизонтальной скорости (2) линейно зависят от координаты $x$, что позволяет описывать течения вязкой несжимаемой жидкости, индуцируемые неоднородными распределениями скоростей (напряжений), температуры и давления на одной или обеих границах бесконечного слоя. Класс решений (2) обобщает известное в гидродинамике точное решение Хименца [27].

Подставим класс точных решений (2) в систему Обербека-Буссинеска (1), получим уравнения:

$$
\begin{gathered}
\left(U+x u_{1}\right) u_{1}+\left(U^{\prime}+x u_{1}^{\prime}\right) w=-P_{1}+v\left(U^{\prime \prime}+x u_{1}^{\prime \prime}\right) \\
w w^{\prime}=-\left(P_{0}^{\prime}+x P_{1}^{\prime}\right)+v w^{\prime \prime}+g \beta\left(T_{0}+x T_{1}\right) \\
\left(U+x u_{1}\right) T_{1}+\left(T_{0}^{\prime}+x T_{1}^{\prime}\right) w=\chi\left(T_{0}^{\prime \prime}+x T_{1}^{\prime \prime}\right) \\
u_{1}+w^{\prime}=0
\end{gathered}
$$

Здесь и далее штрих обозначает производные от функций, входящих в класс (2), по вертикальной (поперечной) координате $z$. Используя метод неопределенных коэффициентов (линейную независимость функций 1 и $x$ ), выпишем отдельно свободные члены и коэффициенты при горизонтальной (продольной) координате $x$ в системе (3):

$$
\begin{gathered}
U u_{1}+U^{\prime} w=-P_{1}+v U^{\prime \prime} ; \\
u_{1}^{2}+u_{1}^{\prime} w=v u_{1}^{\prime \prime} ; \\
w w^{\prime}=-P_{0}^{\prime}+v w^{\prime \prime}+g \beta T_{0} ; \\
-P_{1}^{\prime}+g \beta T_{1}=0 ; \\
U T_{1}+T_{0}^{\prime} w=\chi T_{0}^{\prime \prime} ; \\
u_{1} T_{1}+T_{1}^{\prime} w=\chi T_{1}^{\prime \prime} ;
\end{gathered}
$$




$$
u_{1}+w^{\prime}=0
$$

Полученная нелинейная система обыкновенных дифференциальных уравнений (4)-(10) наследует нелинейные свойства системы (1). Далее рассмотрим случай крупномасштабных конвективных течений вязкой несжимаемой жидкости, для которых можно пренебречь влиянием инерционных эффектов [2]. Такое допущение можно сделать из-за преобладания вязких сил

$$
v\left(\frac{\partial^{2} V_{x}}{\partial x^{2}}+\frac{\partial^{2} V_{x}}{\partial z^{2}}\right)=v\left(U^{\prime \prime}+x u_{1}^{\prime \prime}\right), v\left(\frac{\partial^{2} V_{z}}{\partial x^{2}}+\frac{\partial^{2} V_{z}}{\partial z^{2}}\right)=v w^{\prime \prime}
$$

над инерционными эффектами

$$
V_{x} \frac{\partial V_{x}}{\partial x}+V_{z} \frac{\partial V_{x}}{\partial z}=\left(U+x u_{1}\right) u_{1}+\left(U^{\prime}+x u_{1}^{\prime}\right) w, V_{x} \frac{\partial V_{z}}{\partial x}+V_{z} \frac{\partial V_{z}}{\partial z}=w w^{\prime}
$$

Другими словами, справедливы следующие оценки в силу класса решений (2):

$$
\frac{\left(U+x u_{1}\right) u_{1}+\left(U^{\prime}+x u_{1}^{\prime}\right) w}{v\left(U^{\prime \prime}+x u_{1}^{\prime \prime}\right)}<<1, \frac{w w^{\prime}}{v w^{\prime \prime}}<<1 .
$$

Такая аппроксимация системы уравнений (1) называется приближением Стокса [2] и является наиболее употребительной при исследовании асимптотик нелинейных течений вязкой несжимаемой жидкости [20-23, 25, 26, 28-30]. Далее выясним, при каких безразмерных параметрах справедливо приближение Стокса для системы (4)-(10).

\section{3. Приведение уравнений к безразмерному виду}

Приведем систему уравнений (4)-(10) к безразмерному виду. Для этого выберем следующие масштабные переменные. Горизонтальная координата $x$ характеризуется масштабом $2 l$, а вертикальная координата $z$ - толщиной слоя жидкости $h$. Условие введения двух масштабных переменных длины существенно расширяет классические методы приведения к безразмерным переменным, поскольку позволяет описывать течение жидкости в приближении тонкого слоя $[11,12,19-26]$. Аналогично можно ввести два масштаба для скоростей. Ниже обсудим возможность использовать один базисный масштаб скорости, установив при этом функциональную связь с характерным размером другой компоненты скорости.

Для того чтобы текст статьи не загромождать новыми обозначениями, безразмерные переменные будем обозначать теми же буквами, за исключением координат, а масштабные переменные - в квадратных скобках. В этом случае для однородных слагаемых гидродинамических полей скоростей, давления, температуры, соответственно, масштабные переменные примут следующий вид:

$$
[U],[w],\left[P_{0}\right],\left[T_{0}\right]
$$

Отдельно распишем приведение к безразмерному виду каждого уравнения системы (4)-(10). Будем учитывать размерность дифференциальных операторов для произвольной функции $f$ в следующем виде [2, 34]: 


$$
f^{\prime} \sim \frac{[f]}{h}, f^{\prime \prime} \sim \frac{[f]}{h^{2}} .
$$

Для начала рассмотрим обезразмеривание уравнения (7) и получим зависимость безразмерных величин давления и температуры:

$$
P_{1}^{\prime} \frac{\left[P_{0}\right]}{l h}=g \beta T_{1} \frac{\left[T_{0}\right]}{l} .
$$

Определим размерность давления следующим образом:

$$
\left[P_{0}\right]=g \beta l\left[T_{0}\right] \text {. }
$$

Тогда уравнение (7) запишем в виде:

$$
P_{1}^{\prime}=\delta T_{1}
$$

где $\delta=\frac{h}{l}$ - параметр геометрической анизотропии слоя вязкой несжимаемой жидкости.

Приведем уравнение (4) к безразмерному виду. Получим следующее выражение:

$$
U u_{1}[U] \frac{[U]}{l}+U^{\prime} w \frac{[U]}{h}[w]=-P_{1} \frac{\left[P_{0}\right]}{l}+v U^{\prime \prime} \frac{[U]}{h^{2}} .
$$

Домножим обе части уравнения на число $\frac{[U] l^{2}}{v}$, получим:

$$
U u_{1} \frac{[U] l}{v}+U^{\prime} w \frac{[w] l^{2}}{v h}=-P_{1} \frac{\left[P_{0}\right] l}{v[U]}+v U^{\prime \prime} \frac{l^{2}}{v h^{2}} .
$$

Определим размерность скоростей следующим образом:

$$
\begin{gathered}
{[U]=\frac{\left[P_{0}\right] l}{v}=\frac{g \beta\left[T_{0}\right] l^{2}}{v} ;} \\
{[w]=\frac{\left[P_{0}\right] h}{v}=\frac{g \beta\left[T_{0}\right] l h}{v}=\frac{g \beta\left[T_{0}\right] l^{2} h}{v l} .}
\end{gathered}
$$

Таким образом, получим зависимость введенных безразмерных скоростей в следующем виде:

$$
[w]=\delta[U] .
$$

Подставив введенные размерности в безразмерное первое уравнение системы (4), получим: 


$$
U u_{1} \frac{g \beta\left[T_{0}\right] l^{3}}{v^{2}}+U^{\prime} w \frac{g \beta\left[T_{0}\right] l^{3}}{v^{2}}=-P_{1}+\frac{U^{\prime \prime}}{\delta^{2}}
$$

Используя безразмерные критерии подобия гидродинамики, можно записать

$$
U u_{1} \delta^{2} \mathrm{Gr}+U^{\prime} w \delta^{2} \mathrm{Gr}=-\delta^{2} P_{1}+U^{\prime \prime},
$$

где $\mathrm{Gr}=\frac{g \beta\left[T_{0}\right] l^{3}}{v^{2}}-$ число Грасгофа $[2,34]$. Для рассматриваемой задачи число Грасгофа также можно записать в следующем виде: $\mathrm{Gr}=\frac{[U] l}{v}$ (конвективное число Рейнольдса).

Далее сделаем безразмерным уравнение (10) - уравнение несжимаемости:

$$
u_{1} \frac{[U]}{l}+w^{\prime} \frac{[w]}{h}=0 .
$$

Подставим в это выражение зависимость (12) и получим уравнение в следующем виде:

$$
w^{\prime}+u_{1}=0
$$

Дальше обезразмерим уравнение (5):

$$
u_{1}^{2} \frac{[U][U]}{l^{2}}+u_{1}^{\prime} w \frac{[U]}{l h}[w]=v u_{1}^{\prime \prime} \frac{[U]}{l h^{2}} .
$$

Упростим выражение с учетом зависимости (12) и получим:

$$
u_{1}^{2} \frac{[U]}{l}+u_{1}^{\prime} w \frac{[U]}{l}=v u_{1}^{\prime \prime} \frac{1}{h^{2}}
$$

Умножим полученное выражение на $\frac{l^{2}}{v}$ и получим:

$$
u_{1}^{2} \delta^{2} \mathrm{Gr}+u_{1}^{\prime} w \delta^{2} \mathrm{Gr}=u_{1}^{\prime \prime}
$$

Аналогично обезразмериваем уравнение (6):

$$
w w^{\prime}[w] \frac{[w]}{h}=-P_{0}^{\prime} \frac{\left[P_{0}\right]}{h}+v w^{\prime \prime} \frac{[w]}{h^{2}}+g \beta T_{0}\left[T_{0}\right]
$$


Умножаем обе части уравнения на комплекс $\frac{h^{2}}{v[w]}$ :

$$
w w^{\prime} \frac{[w] h}{v}=-P_{0}^{\prime} \frac{\left[P_{0}\right] h}{v[w]}+w^{\prime \prime}+\frac{g \beta T_{0} h^{2}\left[T_{0}\right]}{v[w]}
$$

Выразим масштабные переменные $[w],\left[P_{0}\right]$ и $\left[T_{0}\right]$ через $[U]$ из выражений (11) и (12) и подставим их в это уравнение. Получим:

$$
w w^{\prime} \frac{\delta[U] h}{v}=-P_{0}^{\prime} \frac{[U] v h}{v l \delta[U]}+w^{\prime \prime}+\frac{g \beta T_{0} h^{2}[U] v}{v \delta[U] g \beta l^{2}} .
$$

Преобразуем выражение и получим окончательный вид:

$$
w w^{\prime} \delta^{2} \mathrm{Gr}=-P_{0}^{\prime}+w^{\prime \prime}+T_{0} \delta .
$$

Следующее обезразмеривание проведем для уравнения (8):

$$
U T_{1}[U] \frac{\left[T_{0}\right]}{l}+T_{0}^{\prime} w \frac{\left[T_{0}\right]}{h}[w]=\chi T_{0}^{\prime \prime} \frac{\left[T_{0}\right]}{h^{2}} .
$$

Упростим выражение и выразим масштабную переменную $[w]$ через $[U]$ из выражения (12) и подставим их в полученное уравнение:

$$
U T_{1} \frac{[U]}{l}+T_{0}^{\prime} w \frac{[U]}{h} \delta=\frac{\chi T_{0}^{\prime \prime}}{h^{2}}
$$

Умножим полученное выражение на $\frac{h^{2}}{v}$ и заменим безразмерным числом Грасгофа:

$$
U T_{1} \delta^{2} \mathrm{Gr}+T_{0}^{\prime} w \delta^{2} \mathrm{Gr}=\frac{T_{0}^{\prime \prime}}{\operatorname{Pr}}
$$

где $\operatorname{Pr}=\frac{v}{\chi}-$ число Прандтля [2, 34].

В последнюю очередь обезразмерим уравнение (9):

$$
u_{1} T_{1} \frac{[U]}{l} \frac{\left[T_{0}\right]}{l}+T_{1}^{\prime} w \frac{\left[T_{0}\right]}{l h}=\chi T_{1}^{\prime \prime \prime} \frac{\left[T_{0}\right]}{l h^{2}}
$$

Проведем преобразования, аналогичные с проведенными для уравнения (8), и получим уравнение:

$$
U T_{1} \delta^{2} \mathrm{Gr}+T_{1}^{\prime} w \delta^{2} \mathrm{Gr}=\frac{T_{1}^{\prime \prime}}{\operatorname{Pr}}
$$


Запишем все безразмерные уравнения в систему:

$$
\begin{gathered}
U u_{1} \delta^{2} \mathrm{Gr}+U^{\prime} w \delta^{2} \mathrm{Gr}=-\delta^{2} P_{1}+U^{\prime \prime} ; \\
u_{1}^{2} \delta^{2} \mathrm{Gr}+u_{1}^{\prime} w \delta^{2} \mathrm{Gr}=u_{1}^{\prime \prime} ; \\
w w^{\prime} \delta^{2} \mathrm{Gr}=-P_{0}^{\prime}+w^{\prime \prime}+T_{0} \delta ; \\
P_{1}^{\prime}=\delta T_{1} ; \\
U T_{1} \delta^{2} \mathrm{Gr}+T_{0}^{\prime} w \delta^{2} \mathrm{Gr}=\frac{T_{0}^{\prime \prime}}{\operatorname{Pr}} \\
U T_{1} \delta^{2} \mathrm{Gr}+T_{1}^{\prime} w \delta^{2} \mathrm{Gr}=\frac{T_{1}^{\prime \prime}}{\operatorname{Pr}} \\
w^{\prime}+u_{1}=0 .
\end{gathered}
$$

В полученной системе (13) будем считать произведение $\delta^{2} \mathrm{Gr}$ малым, в силу выбора приближения тонкого слоя. Следовательно, можно пренебречь слагаемыми, содержащими множитель $\delta^{2} \mathrm{Gr}$ (конвективной производной). В результате получим следующую систему уравнений, записанную в том порядке, в котором будет осуществляться ее интегрирование:

$$
\begin{gathered}
T_{0}^{\prime \prime}=0, T_{1}^{\prime \prime}=0, P_{1}^{\prime}=\delta T_{1} ; \\
U^{\prime \prime}=\delta^{2} P_{1}, u_{1}^{\prime \prime}=0, w^{\prime}+u_{1}=0 ; \\
P_{0}^{\prime}=w^{\prime \prime}+T_{0} \delta .
\end{gathered}
$$

\section{4. Решение краевой задачи}

Последовательное интегрирование систем уравнений (14) позволяет получить общее решение для полей скоростей, температуры и давления, которое имеет вид:

$$
\begin{gathered}
T_{0}=C_{1} z+C_{2}, T_{1}=C_{3} z+C_{4} ; \\
P_{1}=\delta\left(C_{3} \frac{z^{2}}{2}+C_{4} z\right)+C_{5} ; \\
U=\delta^{2}\left[\delta\left(C_{3} \frac{z^{4}}{4 !}+C_{4} \frac{z^{3}}{3 !}\right)+C_{5} \frac{z^{2}}{2}\right]+C_{6} z+C_{7} ; \\
u_{1}=C_{8} z+C_{9}, w=-\left(C_{8} \frac{z^{2}}{2}+C_{9} z\right)+C_{10} ; \\
P_{0}=-C_{8} z-C_{9}+\delta\left(C_{1} \frac{z^{2}}{2}+C_{2} z\right)+C_{11} .
\end{gathered}
$$


Для вычисления частного решения системы (14), описывающего конвекцию вязкой несжимаемой жидкости при задании теплового источника на верхней границе горизонтального слоя, сформулируем граничные условия. Безразмерная толщина слоя жидкости равна единице, а поперечная координата принадлежит множеству $0 \leq z \leq 1$.

На нижней (твердой) границе при $z=0$ выполняются условия прилипания:

$$
V_{x}(0)=V_{z}(0)=0 \text {. }
$$

Следовательно, в силу структуры класса решений (2) условие прилипания записывается следующим образом:

$$
U=w=u_{1}=0 .
$$

На верхней границе $(z=1)$ задано безразмерное неоднородное распределение горизонтальной скорости

$$
V_{x}(1)=W+\Omega x
$$

Следовательно, выполняются равенства:

$$
U(1)=W, u_{1}(1)=\Omega \text {. }
$$

Запишем краевые условия для температуры и давления. Нижняя граница является изотермической, на которой задана отсчетная температура. Без ограничения общности отсчет ведется от нулевого значения:

$$
T_{0}(0)=0, T_{1}(0)=0 \text {. }
$$

На верхней границе задан тепловой источник $V_{x}=A+B x$ и распределено давление:

$$
T_{0}(1)=A, T_{1}(1)=B, P_{0}(1)=S, P_{1}(1)=0 .
$$

Точное решение уравнений (14) с граничными условиями (16)-(19) имеют вид:

$$
\begin{gathered}
T_{0}=A z, T_{1}=B z ; \\
P_{1}=\frac{\delta B}{2}\left(z^{2}-1\right) ; \\
U=\frac{\delta^{3} B z}{4 !}\left(z^{3}-6 z+5\right)+W z ; \\
u_{1}=\Omega z, w=-\frac{\Omega z^{2}}{2} ; \\
P_{0}=\frac{\delta A}{2}\left(z^{2}-1\right)+\Omega(1-z)+S .
\end{gathered}
$$




\section{5. Анализ точного решения}

Проанализируем полученные решения (20). Отметим, что функции $T_{0}$ и $T_{1}$ изменяются по линейному закону с различными коэффициентами пропорциональности, а поведение функций (возрастание или убывание) определяется граничными условиями (рис. 1 a). Такая же ситуация с градиентом давления $P_{1}$ (рис. 1 б). Изолинии температуры, являющие гиперболами, приведены на рис. 2.

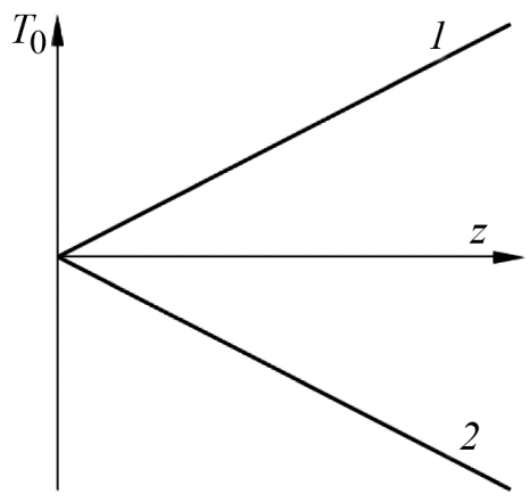

$a$

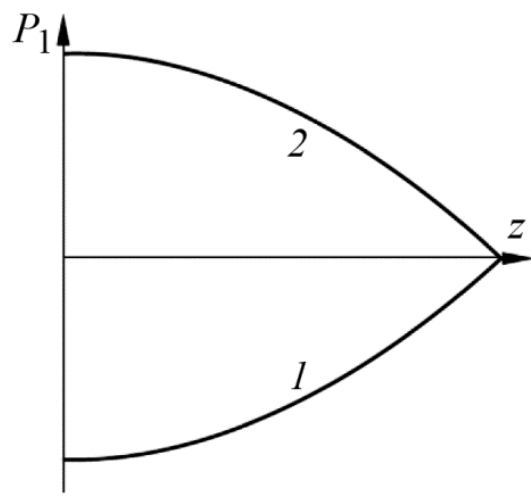

$\sigma$

Рис. 1. Графики: $a-T_{0}$ (прямая $1-A>0$; прямая $2-A<0$ );

$\sigma-P_{1}$ (прямая $1-B>0$; прямая $2-B<0$ ), при различных граничных условиях

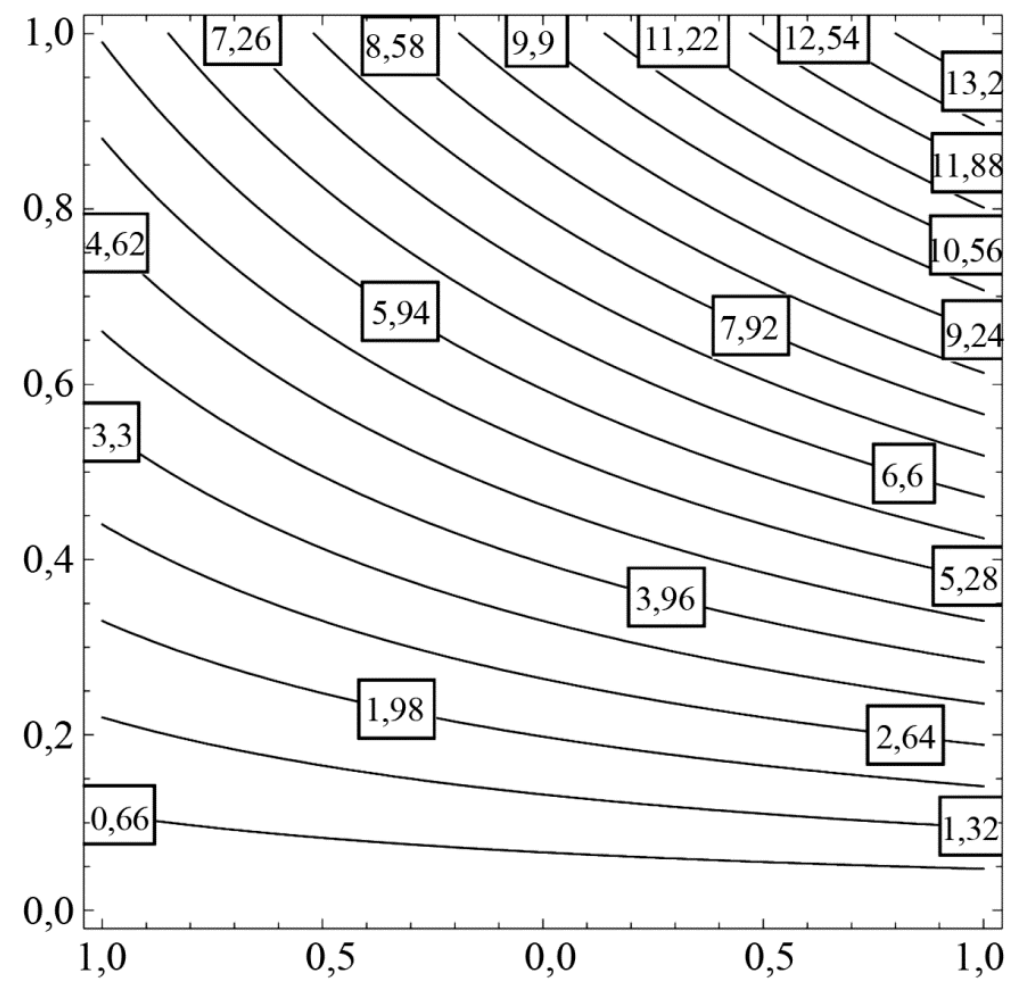

Рис. 2. Изолинии температуры

Скорость $V_{z}$ и составляющая градиента $V_{x}$ по горизонтальной координате не зависят от способа нагрева (положительные или отрицательные значения температуры на границе слоя). При $\Omega>0$ скорость $V_{z}$ монотонно убывает по квадратичному закону по толщине слоя (функция 
$u_{1}$ линейно возрастает). В этом случае жидкость оттекает от верхней границы. Отток жидкости обусловлен растеканием жидкости в горизонтальном направлении (рис. 3 б). При противоположном значении $(\Omega<0)$ наблюдается монотонное возрастание скорости $V_{z}$ ( $u_{1}$ линейно убывает), что говорит об испарении жидкости с верхней границы бесконечного слоя вязкой несжимаемой жидкости (рис. 3 a).
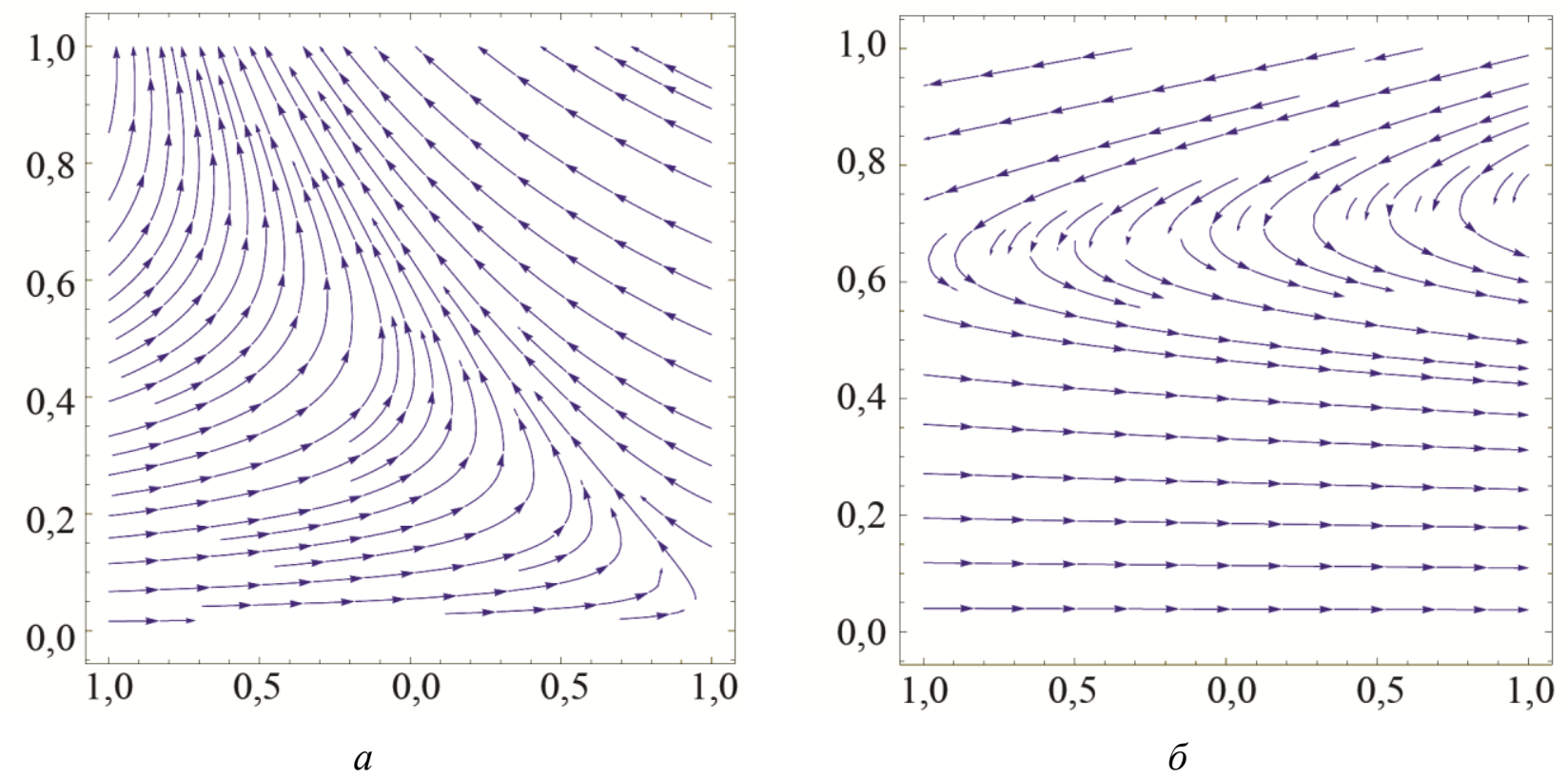

Рис. 3. Линии тока при различных граничных значениях $(a-\Omega<0 ; \sigma-\Omega<0)$

Проанализируем однородную компоненту скорости $U$, параллельную оси абсцисс. Запишем функцию $U$ в следующем виде:

$$
U=z\left[\frac{\delta^{3} B}{4 !}\left(z^{3}-6 z+5\right)+W\right]
$$

При $W=0$ функция $U$ принимает вид:

$$
U=\frac{\delta^{3} B}{4 !}\left[z(z-1)\left(z^{2}+z-5\right)\right]
$$

Данная функция имеет два нуля на области определения, которые являются граничными точками: $z=0$ и $z=1$. Очевидно, что во внутренних точках слоя жидкости скорость сонаправлена оси абсцисс, принимая максимальное значение $U=1,07607 \frac{\delta^{3} B}{24}$ для $z=0,446298$. В том случае, если $W=0$, то $U$ принимает только положительные значения внутри слоя, а нулевое значение имеет только на твердой границе (условие прилипания). Если задать скорость, направление которой противоположно оси абсцисс $(W<0)$, могут существовать застойные точки (скорость принимает нулевые значения). Анализ функции $U$ показывает, что возможна только одна точка, в которой скорость может обратиться в нуль во внутренних точках области определения $U$. Реализация этого случая определяется неравенством 


$$
\frac{5 \delta^{3} B}{24}+W>0
$$

В этом случае скорость $U$ имеет застойную точку и принимает как положительные, так и отрицательные значения, достигая при этом максимального значения внутри слоя.

При выполнении противоположного неравенства скорость $U$ будет отрицательна, монотонно убывая на всей области определения (минимум достигается на верхней границе) (рис. 4 б).

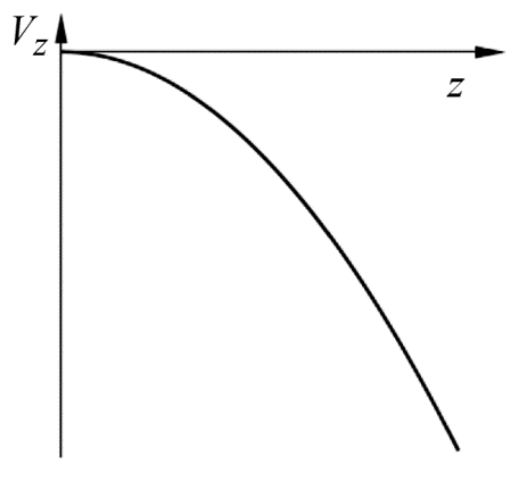

$a$

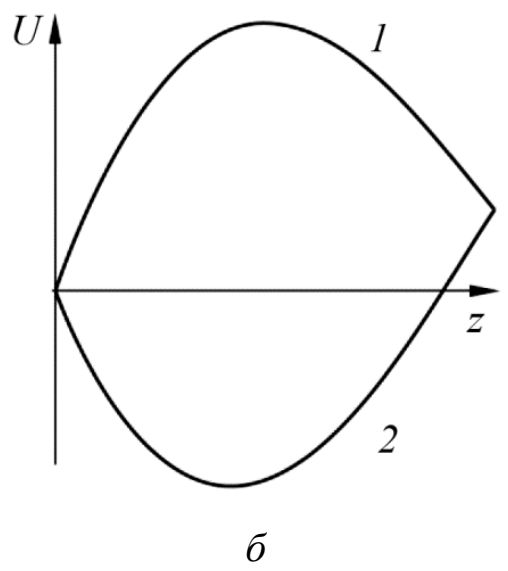

б

Рис. 4. График функций компонент скорости $V_{z}$ и $U$ при различных граничных условиях

Завершаем исследование решений, описывающих конвективное течение КуэттаХименца при линейном распределении температуры на верхней границе, анализом приведенного давления $P_{0}$. При выполнении неравенства

$$
S<\frac{\delta A}{2}-\Omega
$$

давление, отнесенное к плотности, может принимать нулевое значение внутри слоя жидкости. Следовательно, могут существовать при подходящем выборе граничных условий области положительного и отрицательного давления. Отметим, что давление на недеформируемой (нижней) границе может быть меньше, чем на верхней границе. Это возможно при выполнении неравенства

$$
\Omega<\frac{\delta A}{2}
$$

Если строго выполняется противоположное неравенство, то давление на нижней границе (как и в случае гидростатического равновесия) будет больше, чем на свободной поверхности. При выполнении равенства давление принимает одинаковые значения на границе, а внутри слоя оно одного знака (положительное). График функции $P_{0}$ показан на рис. 5 . Изолинии давления показаны на рис. 6. 


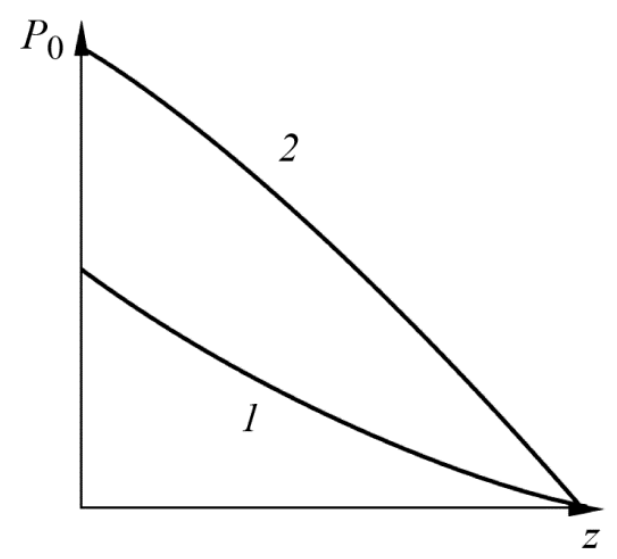

Рис. 5. График функции $P_{0}$ (прямая $1-A>0$; прямая $2-A<0$ )

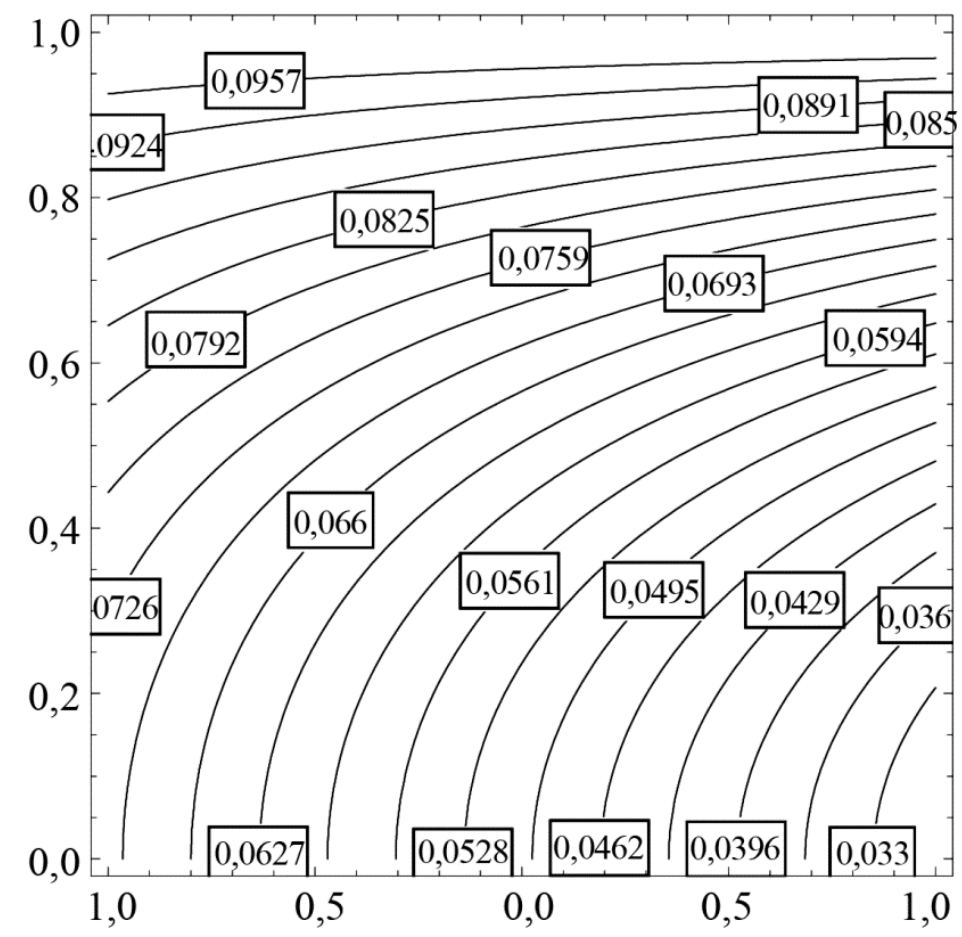

Рис. 6. Изолинии давления

Исследование изобар показывает, что они всегда имеют бесконечно удаленную точку (являются локально гиперболическими или параболическими). Невозможность существования замкнутых изолиний давления обусловлена структурой рассматриваемого точного решения (2) - линейностью поля давления от горизонтальной координаты.

\section{6. Заключение}

В настоящей статье получено обобщение установившегося классического решения Куэтта для неизотермических вязких несжимаемых жидкостей. Изучен линейный случай распределения температуры на свободной границе. Показано существование «застойных» точек у гидродинамических полей при определенных ограничениях на физические постоянные и граничные условия. Посредством методов локализации корней у полиномиальных решений изучены качественные и количественные свойства полученных точных решений в классе линейно растущих скоростей по горизонтальным координатам. 


\section{Литература}

1. Couette M. Etudes sur le frottement des liquides // Ann. Chim. Phys. - 1890. - Vol. 21 P. 433-510.

2. Ландау Л. Д., Лифшиц Е. М. Теоретическая физика : в 10 т. - Т. 6. Гидродинамика, 5-е изд. - М. : Физматлит, 2006. - 736 с.

3. Drazin P. G. The Navier-Stokes equations: A classification of flows and exact solutions. Cambridge : Cambridge Univ. Press, 2006. - 196 p.

4. Boronin S. A. Stability of the plane Couette flow of a disperse medium with a finite volume fraction of the particles // Fluid Dynamics. - 2011. - Vol. 46. - P. 64-71. DOI: $10.1134 / \mathrm{S} 0015462811010078$.

5. Georgiyevskii D. V. Generalized Joseph Estimates of Stability of Plane Shear Flows with Scalar Nonlinearity // Bulletin of the Russian Academy of Sciences: Physics. - 2011. - Vol. 75, no. 1. - P. 149-152. - DOI: 10.3103/S1062873810121044.

6. Zhuk V. I., Protsenko I. G. Asymptotic model for the evolution of perturbations in the plane Couette-Poiseuille flow // Doklady Mathematics. - 2006. - Vol. 74, no. 3. - P. 896-900. DOI: $10.1134 / \mathrm{S} 1064562406060287$.

7. Rudyak V. Ya., Isakov E. B., Bord E.G. Instability of plane Couette flow of two-phase liquids // Technical Physics Letters. - 1998. - Vol. 24. - P. 199-200. - DOI: 10.1134/1.1262051.

8. Troshkin O. V. Nonlinear stability of Couette, Poiseuille and Kolmogorov plane channel flows // Doklady Mathematics. - 2012. - Vol. 85, no. 2. - P. 181-185. - DOI: 10.1134/S1064562412020068.

9. Shalybkov D. A. Hydrodynamic and hydromagnetic stability of the Couette flow // PhysicsUspekhi. - 2009. - Vol. 52, no. 9. - P. 915-935. - DOI: 10.3367/UFNe.0179.200909d.0971.

10. Абрамян А. К., Миранцев Л. В., Кучмин А. Ю. Моделирование течения Куэтта простой жидкости в плоском канале наноразмерной высоты // Математическое моделирование. 2012. - T. 24, № 4. - C. 3-21.

11. Aristov S. N., Prosviryakov E. Yu. Nonuniform convective Couette flow // Fluid Dynamics. 2016. - Vol. 51. - P. 581-587. - DOI: 10.1134/S001546281605001X.

12. Aristov S. N., Prosviryakov E. Yu. A new class of exact solutions for three-dimensional thermal diffusion equations // Theor. Found. Chem. Eng. - 2016. - Vol. 50, no. 3. - P. 286-293. DOI: $10.1134 / \mathrm{S} 0040579516030027$.

13. Babkin V. A. Plane Turbulent Couette Flow // Journal of Engineering Physics and Thermophysics. - Vol. 76, iss. 6. - P. 1251-1254. - DOI: 10.1023/B:JOEP.0000012026.19646.c6.

14. Беляева Н. А., Кузнецов К. П. Анализ нелинейной динамической модели течения Куэтта структурированной жидкости в плоском зазоре // Вестн. Сам. гос. техн. ун-та. Сер. Физ.-мат. науки. - 2012. - № 2 (27). - C. 85-92. - DOI: 10.14498/vsgtu1018.

15. Gavrilenko S. L., Shil'ko S. V., Vasin R. A. Characteristics of a viscoplastic material in the couette flow // Journal of Applied Mechanics and Technical Physics. - 2002. - Vol. 43, iss. 3. P. 439-444. - DOI: 10.1023/A:1015378622918.

16. Кудинов В.А., Кудинов И.В. Получение точных аналитических решений гиперболических уравнений движения при разгонном течении Куэтта // Известия Российской академии наук. Энергетика. - 2012. - № 1. - С. 119-133.

17. Malyshev V. A., Manita A. D. Stochastic Micromodel of the Couette Flow // Theory of Probability \& Its Applications. - 2009. - Vol. 53, iss. 4. - P. 716-727. - DOI: 10.1137/S0040585X97983924.

18. Pukhnachev V. V., Pukhnacheva T. P. Couette Problem for Kelvin-Voigt Medium // Journal of Mathematical Sciences. - 2012. - Vol. 186, iss. 3. - P. 495-510. - DOI: 10.1007/s10958-012-1003-0.

19. Скульский О. И., Аристов С. Н. Механика аномально вязких жидкостей. - МоскваИжевск : НИЦ «Регулярная и хаотическая динамика», 2003. - 156 с.

20. Aristov S. N., Privalova V. V., Prosviryakov E. Y. Stationary nonisothermal Couette flow. Quadratic heating of the upper boundary of the fluid layer // Russian Journal of Nonlinear Dynamics. - 2016. - Vol. 12, no. 2. - P. 167-178. - DOI: 10.20537/nd1602001. 
21. Аристов С. Н., Просвиряков Е. Ю. Об одном классе аналитических решений стационарной осесимметричной конвекции Бенара-Марангони вязкой несжимаемой жидкости // Вестн. Сам. гос. техн. ун-та. Сер. Физ.-мат. науки. - 2013. - № 3 (32). - С. 110-118.

22. Аристов С. Н., Просвиряков Е. Ю. Точные решения термокапиллярной конвекции при локализованном нагреве плоского слоя вязкой несжимаемой жидкости // Вестн. Казан. гос. техн. ун-та им. А. Н. Туполева. - 2014. - № 3. - С. 7-12.

23. Аристов С. Н., Власова С. С., Просвиряков Е. Ю. Линейная конвекция БенараМарангони при квадратичном нагреве сверху плоского слоя вязкой несжимаемой жидкости // Ползуновский вестник. - 2014. - № 4-2. - С. 95-102.

24. Aristov S. N., Shvarts K. G. Convective heat transfer in a locally heated plane incompressible fluid layer // Fluid Dynamics. - 2013. - Vol. 48. - P. 330-335. - DOI: 10.1134/S001546281303006X.

25. Vlasova S. S., Prosviryakov E. Y. Parabolic convective motion of a fluid cooled from below with the heat exchange at the free boundary // Russian Aeronautics. - 2016. - Vol. 59, no. 4. P. 529-535. - DOI: 10.3103/S1068799816040140.

26. Vlasova S. S., Prosviryakov E. Yu. Two-dimensional convection of an incompressible viscous fluid with the heat exchange on the free border // Vestn. Samar. Gos. Tekh. Univ., Ser. Fiz.-Mat. Nauki. - 2016. - Vol. 20, no. 3. - P. 567-577. - DOI: 10.14498/vsgtu1483.

27. Hiemenz K. Die Grenzschicht an einem in den gleichförmigen Flüssigkeit-sstrom eingetauchten geraden Kreiszylinder // Dingler's Politech. J. - 1911. - Vol. 326. - P. 321-324.

28. Andreev V. K., Cheremnykh E. N. 2D thermocapillary motion of three fluids in a flat channel. Journal of Siberian Federal University // Mathematics and Physics. - 2016. - Vol. 9, iss. 4. P. 404-415. - DOI: 10.17516/1997-1397-2016-9-4-404-415.

29. Andreev V. K., Cheremnykh E. N. The joint creeping motion of three viscid liquids in a plane layer: A priori estimates and convergence to steady flow // Journal of Applied and Industrial Mathematics. - 2016. - Vol. 10, no. 1. - P. 7-20. - DOI: 10.1134/S1990478916010026.

30. Андреев В. К., Собачкина Н. Л. Движение бинарной смеси в плоских и цилиндрических областях. - Красноярск : СФУ, 2012. - 187 с. - ISBN 978-5-7638-2372-1.

31. Aristov S. N., Knyazev D. V., Polyanin A. D. Exact solutions of the Navier-Stokes equations with the linear dependence of velocity components on two space variables // Theoretical Foundations of Chemical Engineering. - 2009. - Vol. 43, no. 5. - P. 642-662. DOI: 10.1134/S0040579509050066.

32. Pukhnachev V. V. Group Properties of the Navier-Stokes Equations in a Plane Case // Prikl. Mekh. Tekh. Fiz. - 1960. - No. 1. - P. 83-90.

33. Ekman V. W. On the Influence of the Earth's Rotation on Ocean-Currents // Ark. Mat. Astron. Fys. - 1905. - Vol. 2, no. 11. - P. 1-52.

34. Гершуни Г. З., Жуховицкий Е. М. Конвективная устойчивость несжимаемой жидкости. - Москва : Наука, 1972. - 392 с.

35. Современные математические модели конвекции / В. К. Андреев, Ю. А. Гапоненко, О. Н. Гончарова, В. В. Пухначев - М. : ФИЗМАТЛИТ, 2008. - 368 с.

36. Sidorov A. F. Two classes of solutions of the fluid and gas mechanics equations and their connection to traveling wave theory // J. Appl. Mech. Tech. Phys. - 1989. - Vol. 30, no. 2. P. 197-203. - DOI: 10.1007/BF00852164.

37. Lin C. C. Note on a class of exact solutions in magneto-hydrodynamics // Arch. Rational Mech. Anal. - 1958. - Vol. 1. - P. 391-395. 